\title{
The impact of Angiotensin II Type 1 Receptor antibodies on morbidity and mortality in Heart Mate II supported recipients
}

\author{
Marian Urban ${ }^{\mathrm{a}}$, Antonij Slavcev ${ }^{\mathrm{b}}$, Tomas Gazdic, Peter Ivaka, Ivan Netuka ${ }^{\mathrm{a}}$
}

\begin{abstract}
Aims. One of the proposed limitations of left ventricular assist device (LVAD) therapy is high degree of sensitization. Apart from human leukocyte antigen (HLA), antibodies against Angiotensin II Type 1 Receptor (AT1R) have been associated with adverse outcomes. The purpose of this study was to compare complications and survival of anti - AT1R positive versus negative Heart Mate II (HMII) recipients.

Methods. Altogether 96 patients received HMII at our institution between 2008 and 2012. These were stratified into three groups: antibody positive before implantation (AT1R+), antibody conversion during support (AT1R-/+) and patients who remained antibody negative (AT1R-). Survival, major on-device adverse events and post-transplant rejections were assessed with Kaplan-Meier and log-rank tests.

Results. Two year on-device and overall survival was $78 \pm 12 \%$ and $75 \pm 10 \%$ in AT1R-, $60 \pm 23 \%$ and $60 \pm 15 \%$ in AT1R+ and $92 \pm 6 \%$ and $87 \pm 5 \%$ in AT1R-/+ group $(P=0.409, P=0.185)$. Freedom from major adverse event at two years for AT1R-, AT1R+ and AT1R-/+ was $49 \pm 14 \%, 53 \pm 16 \%$ and $41 \pm 11 \%(P=0.875)$. Freedom from rejection was $63 \pm 17 \%$ in patients who were both anti-AT1R and HLA negative and $65 \pm 13 \%$ in those who were antibody positive $(P=0.788)$.

Conclusion. Patients who were anti-AT1R antibody positive had similar on-device survival and rate of complications in comparison to those who were antibody negative. In transplanted patients, there were no differences in the overall survival and rejection between the groups.
\end{abstract}

Key words: Heart Mate II, LVAD, Angiotensin II Type 1 Receptor, heart transplantation

Received: February 1, 2016; Accepted: April 19, 2016; Available online: April 27, 2016

http://dx.doi.org/10.5507/bp.2016.025

${ }^{a}$ Department of Cardiac Surgery, Institute for Clinical and Experimental Medicine, Prague, Czech Republic

${ }^{b}$ Department of Clinical Immunology, Institute for Clinical and Experimental Medicine, Prague, Czech Republic

'Department of Cardiology, Institute for Clinical and Experimental Medicine, Prague, Czech Republic

Corresponding author: Marian Urban, e-mail:murban@doctors.org.uk

\section{INTRODUCTION}

Left ventricular assist devices (LVAD) reduce heart transplant waiting list mortality and improve the quality of life and survival in selected group of patients with end - stage heart failure ${ }^{1}$. One of the proposed limitations of mechanical support therapy is a higher degree of sensitization among LVAD recipients. Apart from antibodies directed against human leukocyte antigen (HLA), several non-HLA antibodies such as autoantibodies against Angiotensin II type 1 receptor (AT1R) have been associated with an LVAD use ${ }^{2}$. AT1R differs from all other non-HLA antigenic targets in the mechanism of action. The binding of antibodies to AT1R induces physiological effects that mimic those of natural ligand in the reninAngiotensin system ${ }^{3}$. Anti-AT1R antibodies exert their damaging effect by binding to the second extracellular loop of AT1R receptors present in endothelial and vascular smooth muscle cells, inducing endothelial activation and dysfunction. Previous reports have identified heart transplant recipients who developed anti-AT1R antibodies to be at increased risk of post-transplant rejection and cardiac allograft vasculopathy ${ }^{4,5}$. Apart from an effect on the vascular tone, these antibodies also lead to pro - inflammatory and pro - coagulatory responses. The objectives of our study were to evaluate the degree of sensitization against AT1R among our LVAD recipients and also to assess whether the presence of these antibodies could cause a higher incidence of thromboembolic and infectious complications.

\section{MATERIALS AND METHODS}

\section{Patients}

We prospectively evaluated the presence of anti-AT1R antibodies in 96 consecutive Heart Mate II recipients at our institution between 2008 and 2012. After excluding 13 patients who died within 60 days of implantation, 83 patients with a mean duration of $375 \pm 34$ days of support were left for the analysis. Out of a total of 83 patients, 69 eventually underwent heart transplantation, 9 died on support, three were explanted for recovery and two were still alive on support at the last day of follow-up. Follow-up of all transplanted patients ended on 5 April 2015, was $100 \%$ complete, and totalled 2587 patient-months.

\section{Antibody Analysis}

Serum samples were collected before implanting the device and at the pre-determined time points throughout the 
support. Anti-AT1R antibodies were assayed by sandwich enzyme-linked immunosorbent assay (ELISA) using a commercially available kit (CellTrend, Luckenwalde, Germany).

Peripheral blood was obtained into sterile $10-\mathrm{mL}$ serum separator tubes. Samples were centrifugated at $1000 \mathrm{~g}$ for $15 \mathrm{~min}$; serum was collected and stored at $20{ }^{\circ} \mathrm{C}$ until the day of measurement. The concentration of anti-AT1R IgG antibody in serum was measured by ELISA according to the manufacturer's instructions. The samples were assayed on Angiotensin II type 1-receptorprecoated microtiter plates. Standards and diluted 1:100 samples were added into the wells and incubated for two hours at $2-8{ }^{\circ} \mathrm{C}$. After washing steps, anti-AT1R antibody was detected with POD-labelled anti-human IgG antibody (1:100) followed by color development with TMB substrate solution and, measured at $450 \mathrm{~nm}$, with correction wavelength set at $630 \mathrm{~nm}$. Optical densities were then converted into concentration by standard curve. The detection range of the test was $>2,5 \mathrm{U} / \mathrm{mL}$ with positive value set at $17 \mathrm{U} / \mathrm{mL}$ and negative $\leq 17 \mathrm{U} / \mathrm{mL}$.

\section{Adverse Events Definitions}

Various adverse clinical events during the support were compared between antibody positive and antibody negative recipients. Standard INTERMACS definitions were used to classify individual post Heart Mate II implantation adverse events ${ }^{6}$.

\section{Statistical Analysis}

Continuous variables are presented as median with 25 th and 75 th percentile interval. Categorical variables are shown as the percentages. The $\chi 2$-test and Fisher's exact test were used to evaluate categorical variables. The data were analysed using the Mann-Whitney U test and Kruskal Wallis one - way analysis of variance for multiple group analysis. Survival and time-to-event analyses were assessed by Kaplan-Meier method and the log-rank test was used for comparisons. Heart Mate II recipients were censored for transplantation and LVAD explantation after recovery to calculate estimated on-device survival. For overall survival analysis, all patients were censored on the date of death or at conclusion of the study. Only patients surviving the first 60 days post Heart Mate II implantation were included in the on-device survival analysis. Date of Heart Mate II implantation was set as the time origin for survival and freedom from LVAD associated adverse event analyses and the date of transplantation as the time origin for freedom from rejection analysis. The linearized rate for each adverse event was calculated as total number of observed events divided by total patient-years of follow-up and expressed as episodes per one patient year (eppy). A $P$ value $<0.05$ was considered significant. The statistical analyses were performed with IBM SPSS 18 (SPSS Inc., Chicago, Il, USA).

Table 1. Basic characteristics of AT1R antibody negative versus positive HeartMate II recipients before implantation.

\begin{tabular}{lccc}
\hline & AT1R positive $(\mathrm{n}=13)$ & AT1R negative $(\mathrm{n}=70)$ & $P$ \\
\hline Age, years & $50(40,59)$ & $45(33,58)$ & 0.607 \\
BMI & $25.4(22.9,27.8)$ & $22.6(20.3,25.9)$ & 0.021 \\
Male gender, \% & $11(85)$ & $60(86)$ & 0.918 \\
Ischemic etiology of heart failure, \% & $3(23)$ & $24(34)$ & 0.766 \\
HLA sensitized, \% & 0 & $4(6)$ & 0.477 \\
Previous mechanical support, \% & $2(14)$ & $8(11)$ & 0.822 \\
Previous sternotomy, \% & $2(14)$ & $15(21)$ & 0.660 \\
\hline
\end{tabular}

BMI, body mass index; HLA, human leukocyte antigen

Table 2. Comparison of AT1R negative patients versus those who became AT1R positive during HeartMate II support.

\begin{tabular}{lccc}
\hline & AT1R negative $(\mathrm{n}=20)$ & AT1R positive $(\mathrm{n}=50)$ & $P$ \\
\hline Age, years & $47(41,57)$ & $51(36,59)$ & 0.969 \\
BMI & $26.5(23.3,28.8)$ & $25.0(22.0,27.0)$ & 0.326 \\
HMII duration of support, days & $324(137,470)$ & $246(129,416)$ & 0.907 \\
PRBC during implantation, units & $9(6,18)$ & $10(8,14)$ & 0.608 \\
FFP, units & $26(15,32)$ & $26(22,34)$ & 0.856 \\
Platelets, units & $3(2,4)$ & $4(3,6)$ & 0.277 \\
Ischemic etiology of heart failure, $\%$ & $6(30)$ & $18(36)$ & 0.696 \\
Previous mechanical support, \% & $1(5)$ & $6(12)$ & 0.730 \\
Previous sternotomy, \% & $5(25)$ & $10(20)$ & 0.800 \\
HLA sensitized, \% & $8(40)$ & $15(30)$ & 0.545 \\
Male gender, \% & $18(90)$ & $42(84)$ & 0.713 \\
Driveline infection, \% & $4(20)$ & $13(26)$ & 0.761
\end{tabular}

BMI, body mass index; HMII, Heart Mate II; PRBC, pure red blood cells; FFP, fresh frozen plasma; HLA, human leukocyte antigen 


\section{RESULTS}

Anti-AT1R antibodies were observed in 13/83 (16\%) of the recipients before Heart Mate II implantation (Table $1)$. Four of these patients (6\%) were also sensitized against HLA antigens. During the support, 50 patients (71\%) who were initially anti-AT1R negative became positive (AT1R-/+) and 20 (29\%) remained negative (AT1R-). Total amount of Heart Mate II support for all 83 patients was 86.7 patient-years. There were no differences in the duration of support or the amount of the blood products used between LVAD recipients who remained negative and those who became positive. Basic demographic and clinical characteristics of both patients groups are summarized in Table 2. Out of 20 patients who remained negative on the mechanical device, 8 became sensitized to HLA antigens. In a cohort of 50 LVAD recipients who developed anti - AT1R antibodies during the support, 15 recipients also developed concurrent anti - HLA antibodies.

\section{Survival}

Out of 83 LVAD recipients who survived 60 days post-implantation, 9 additional patients died after a mean duration of support of 462 (minimum 82, maximum 1123) days. Two year estimated on - device survival was $78 \pm 12 \%$ in AT1R-, $60 \pm 23 \%$ in AT1R+ and $92 \pm 6 \%$ in AT1R-/+ group $(P=0.409)$ (Fig. 1). Overall survival for AT1R-, AT1R+ and AT1R-/+ was $75 \pm 10 \%, 60 \pm 15 \%$ and $87 \pm 5 \%$ at two years and $70 \pm 10 \%, 60 \pm 15 \%$ and 82 $\pm 6 \%$ at four years from Heart Mate II implantation $(P=$ 0.185) (Fig. 2).

\section{Major adverse events}

Freedom from device malfunction, major infection, major bleeding and neurologic dysfunction at two years for AT1R-, AT1R+ and AT1R-/+ was $49 \pm 14 \%, 53 \pm 16 \%$ and $41 \pm 11 \%(P=0.875)$ (Fig. 3$)$.

\section{Device malfunction}

Altogether 5 patients (6\%) experienced device malfunction in our cohort (0.06 eppy). All episodes were related to pump failure (pump thrombosis in four patients and kinked outflow graft in one patient) and resulted in pump exchange in two patients and death in two patients. One patient with pump thrombosis was successfully treated conservatively and subsequently transplanted. Freedom from device malfunction at 2 years in AT1R+, AT1R- and AT1R-/+ was $100 \%, 95 \pm 5 \%$ and $86 \pm 8 \%(P=0.487)$.

\section{Major bleeding}

Our institutional protocol for patients supported with HeartMate II device is anticoagulation with Warfarin (target INR of $1.8-2.2$ ) without antiplatelet therapy. Out of 83 , three patients (4\%) experienced major bleeding episode after 7 days post implantation ( 0.03 eppy). The reasons for readmissions for bleeding were epistaxis, retroperitoneal bleeding and GI bleeding. All patients were discharged home following their bleeding episode

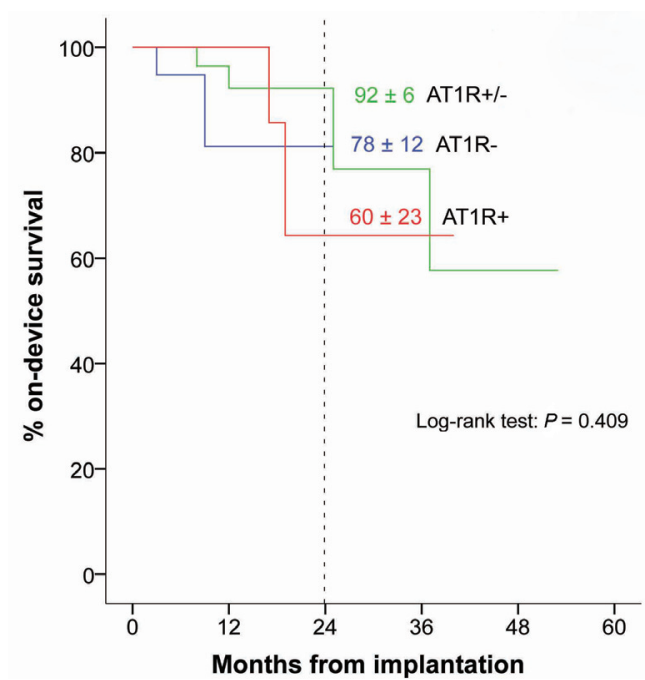

Fig.1. On-device survival of HeartMate II recipients stratified according the presence of anti-AT1R antibodies.

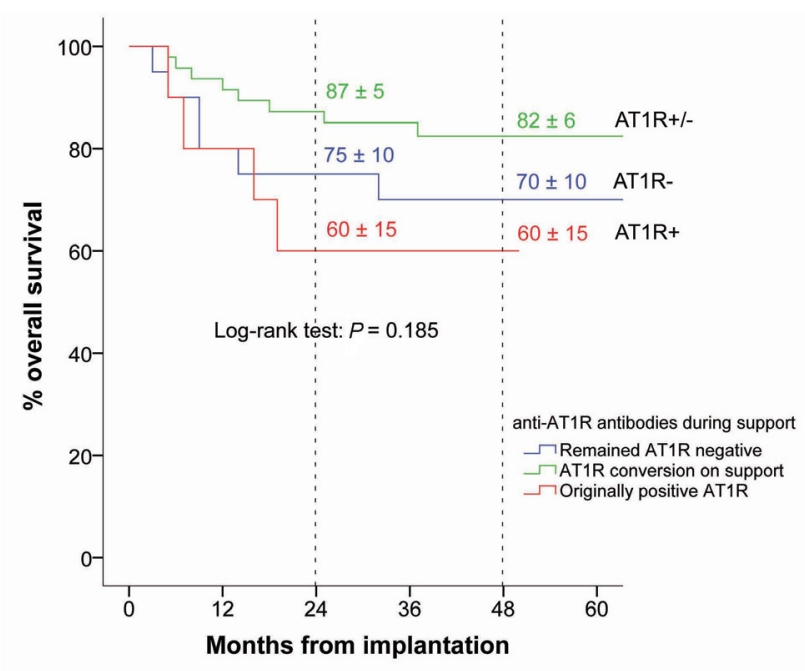

Fig. 2. Overall survival of HeartMate II recipients stratified according the presence of anti-AT1R antibodies.

and all three eventually underwent heart transplantation. Freedom from major bleeding at 2 years in AT1R+, AT1Rand AT1R-/+ was $100 \%, 100 \%$ and $90 \pm 5 \%(P=0.232)$.

\section{Major infection}

More than one third (27 patients, 33\%) of our patients were readmitted due to infection during the course of their mechanical support $(0.3)$. These patients fell into two major categories: infection of a drive - line site (21 patients) and deep sternal wound infection (6 patients). Two patients experienced both drive - line and deep sternal wound infections. One patient with deep sternal wound infection developed sepsis, multi - organ failure and subsequently died as a direct consequence of LVAD infection. Freedom from major infection at 2 years in AT1R+, AT1R- and AT1R-/+ was $54 \pm 16 \%, 62 \pm 13 \%$ and $51 \pm$ $11 \%(P=0.594)$. 


\section{Neurological dysfunction}

Altogether six (7\%) patients experienced neurological dysfunction. Four patients suffered from hemorrhagic CVA (0.05 eppy) and two from ischemic CVA (0.02 eppy). Two of the patients recovered and were subsequently transplanted, four died as a result of CVA. Freedom from neurologic dysfunction at 2 years in AT1R+, AT1Rand AT1R-/+ was $87 \pm 12 \%, 93 \pm 7 \%$ and $92 \pm 6 \%(P=$ 0.997).

\section{Post transplantation rejection}

Out of 69 transplanted patients 8 did not survive to discharge and had no biopsy results available. Of the 61 transplant survivors, 44 patients were anti - AT1R positive and 17 were anti - AT1R antibody negative at the time of transplant. There was no difference in freedom from rejection $(A C R \geq 2 R$ and/or $p A M R \geq 1$ ) among transplant survivors based on the pre-transplant presence of anti-HLA and anti-AT1R antibodies (Fig. 4).

\section{DISCUSSION}

Left ventricular assist devices are a recognized risk factor for sensitization of patients awaiting cardiac transplantation $^{7-9}$. The negative impact of anti - HLA antibodies on post - transplant allograft function and survival has now been well documented. Recently, there has been accumulating evidence of various non - HLA antibodies involvement in decreased allograft and recipient survival ${ }^{5-10}$. While anti - HLA antibodies exert their negative effect via complement activation and antibody - mediated cytotoxicity, antibodies against AT1R, act as a natural allosteric receptor agonist. Angiotensin type 1 receptor is a $\mathrm{G}$ protein-coupled receptor (GPCR) that mediates physiologic actions of Angiotensin II. Binding of agonistic antibodies to AT1R causes activation of the phosphatidylinositolcalcium second messenger system, phosphorylation of extracellular signal-regulated kinase 1/2 (Erk 1/2), activator protein 1 (AP-1) activation, and increase DNA-binding activity of nuclear factor- $\mathrm{B}$ (NF-kB) pro-inflammatory target genes ${ }^{11}$. Anti-AT1R antibodies also trigger tissue factor induction, as evidenced by intense diffuse tissue staining of epithelial, endothelial and mesangial cells in the renal transplant biopsy specimens obtained at the time of AT1R antibody mediated rejection in the absence of complement activation ${ }^{3}$. Anti-AT1R antibodies derived from preeclamptic patients enhanced promoter activity of tissue factor, an initiator of extrinsic coagulation pathway and a target gene for AP- 1 and NF-kB in vitro ${ }^{12}$. AntiAT1R antibodies developed during pregnancy cause both maternal and fetal pathology via pro-inflammatory, vasoconstrictive, pro-coagulatory and pro-apoptotic actions on the placenta ${ }^{13}$. There is also evidence that anti-AT1R antibodies promote endothelial micro particles formation through activating p38 mitogen-activated protein kinase pathway. The "injured" endothelial micro particles trigger reactive oxygen species production and reduce nitric oxide synthesis in vitro experiments ${ }^{14}$. Zhang et al. ${ }^{15}$ investigated in an animal model the association between autoantibod-

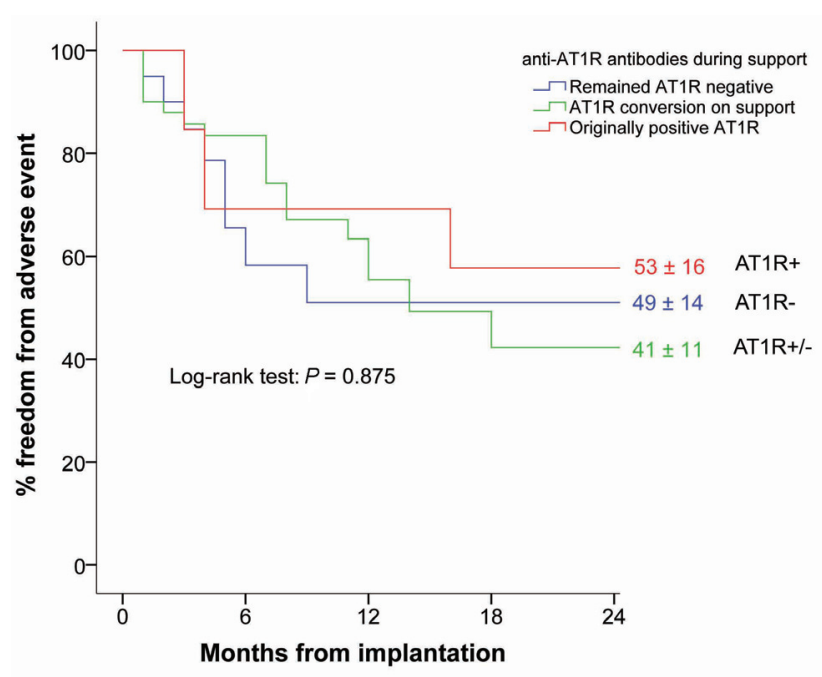

Fig. 3. Freedom from Heart Mate II post - implantation adverse events (device malfunction, major bleeding, major infection and neurologic dysfunction).

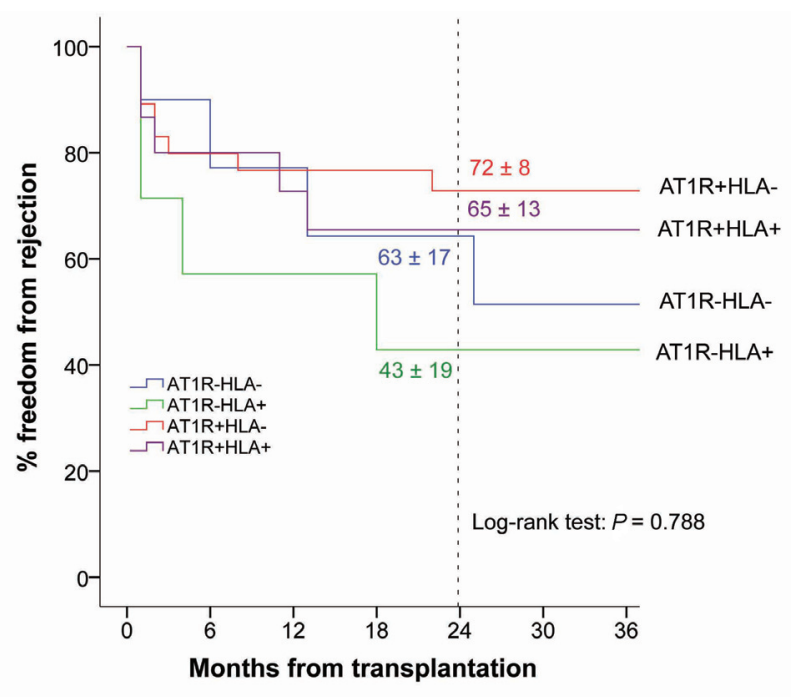

Fig. 4. Freedom from rejection of transplanted patients stratified according the presence of anti-AT1R antibodies and antiHLA antibodies.

ies against AT1 receptor and endothelial dysfunction in vivo. The investigators demonstrated an increased activity of lactate dehydrogenase (LDH) in anti-AT1R positive rats which was regarded as an indicator of cell necrotic death. Functional assessment revealed a decline in the endothelium - dependent relaxation and up - regulation of endothelial intracellular adhesion molecule - 1 (ICAM-1) suggesting that endothelial cells may have inflammatory lesions in anti-AT1R positive rats. Given the known potential of these antibodies to activate inflammatory and coagulation cascade we hypothesized that mechanically bridged patients with raised levels of anti - AT1R antibodies may experience increased rate of thromboembolic and infectious complications while on support.

Our results showed that $16 \%$ of our patients with end - stage heart-failure were already anti - AT1R positive before LVAD implantation. This finding is in agreement 
with Du et al. ${ }^{16}$, who showed that anti-AT1R antibodies exist in the sera of congestive heart failure patients with ischemic cardiomyopathy and hypertension. The authors suggested that these antibodies may play an important role in the pathogenesis and myocardial remodelling of heart failure. Anti-AT1R antibodies develop through similar pathways as those observed for HLA specific antibodies: transfusions, pregnancies and prior transplant. We did not find any association between basic demographic and clinical characteristics (female gender/ previous pregnancy, history of surgery) and sensitization against AT1R before LVAD implantation.

There is accumulating evidence that LVAD support may be associated not only with an increased anti-HLA but also various anti non-HLA antibodies. Hiemann et al. ${ }^{5}$ reported in their pilot study that patients on assist device support before heart transplantation were more likely to develop high anti - AT1R antibody levels (43\% of supported versus $18 \%$ of non - supported patients, $P=$ 0.021 ) within $24 \mathrm{~h}$ after heart transplantation, implicating pre - transplant sensitization. Barten el al. ${ }^{2}$ found in their study of 29 VAD recipients that $65.5 \%$ were positive for anti-AT1R antibodies. Our results confirmed these findings. During the support $71 \%$ of the initially negative AT1R patients became positive. There are multiple pathways by which the production of antibodies against AT1R in patients supported with mechanical devices may be initiated. Protein antigenic determinants from targets may become accessible after injury or surgical stress. Inflammatory events might lead to de novo expression of autoantigens ${ }^{17}$. These autoantibodies are generally of the IgG class requiring T cell help ${ }^{18}$. T cell self-tolerance may be broken by an inflammatory event or hypoxia. We observed no association between pre-operative demographics, blood product peri-operative use or duration of mechanical support and conversion of AT1R negative to AT1R positive status.

Apart from longer waiting times with associated increased morbidity and mortality, there have been no reports linking anti HLA or anti non-HLA antibodies in mechanically bridged recipients to post-LVAD adverse outcomes. Our theory that anti-AT1R antibodies with their proinflamatory and procoagulation properties and their ability to cause endothelial dysfunction may lead to an increased rate of thromboembolic and infectious complications in LVAD recipients was not borne out in our results. There was no difference in the overall survival among patients who were anti-AT1R antibody negative before Heart Mate II implantation and patients who either became positive or remained negative during the support. The incidence of device malfunction, bleeding, infection and neurological dysfunction was not influenced by the presence of anti-AT1R antibodies. There are several possible explanations for the lack of negative impact of AT1R activating antibodies on survival and adverse LVAD related complications in our cohort. Biological impetus regulating At1R antibody injury is fairly complex. Level of AT1R and induction of specific conformations is dependent on individual genetic polymorphisms and the state of local tissue expression influenced by various stressors.
AT1R gene has 14 described polymorphisms, and some of them act as gain or loss of function mutations implicated in receptor activation ${ }^{19}$. The most extensively studied A1166C polymorphism is associated with increased responsiveness to Angiotensin II and various cardiovascular and renal pathologies ${ }^{20}$. It is plausible that mechanical circulatory support with the continuous flow creates a unique microenvironment resulting in lower AT1R expression, potentially less susceptible to anti-AT1R antibody mediated actions. There is compelling evidence that the AT1R may also be activated by mechanical stress without the involvement of Angiotensin II (ref. ${ }^{21}$ ). The AT1R is the first recognized mechanosensitive GPCR ( ref. $^{22}$ ). It is plausible that in the situation when the heart is fully unloaded with mechanical assist device AT1R would be down regulated. There may also be other factors that influence the features of anti-AT1R antibodies, changing their agonistic affinity. The tissue damage caused by certain mechanisms prior to anti-AT1R binding may affect the level of AT1R expression, resulting in different degree of anti-AT1R binding efficiency. Several modifiers have been identified thus far: ischemia, inflammatory events, and microbiome $e^{23,24}$.

\section{Limitations}

Our study has several limitations inherent to the single centre observational study. Due to the small sample size and high correlation between variables, no multivariable models were fitted. Another implication of a small sample size with is a potential for Type II error. To counterbalance relatively small number of adverse events we combined several events into one composite outcome for the time to event analysis.

AT1R gene (located on chromosome 3) has 14 described polymorphisms. We did not perform a genetic analysis of our LVAD recipients and it is conceivable that the differences in expression and activation of AT1R based on genetic mutations could account for variability in AT1R - antibody mediated action.

\section{CONCLUSIONS}

The primary finding of this study is that patients who received a long term LVAD developed a high degree on sensitization against AT1R after implantation. The data showed no impact of anti-AT1R antibodies in Heart Mate II recipients on the overall survival and incidence of LVAD related complications. With the growing population of LVAD supported patients, increasing periods of support times and improved survival, attention is now shifting to the complications of mechanical support. We believe that determining the anti-AT1R antibody profile may prove valuable in risk assessment of mechanically assisted patients and serve as a novel biomarker for the detection of LVAD recipients at risk of an adverse outcome. The impact of anti-AT1R antibodies on the postheart transplantation outcome will have to be evaluated in further studies. 
Author contributions: MU: manuscript writing, literature search, data analysis; AS: literature search; TG: literature search, data analysis; PI: data analysis; IN: final approval. Conflict of interest statement: The authors state that there are no conflicts of interest regarding the publication of this article.

\section{REFERENCES}

1. Trivedi JR, Cheng A, Singh R, Williams ML, Slaughter MS. Surviva on the heart transplant waiting list: impact of continuous flow left ventricular assist device as bridge to transplant. Ann Thorac Surg 2014;98:830-4

2. Barten MJ, Dragun D, von Salisch S, Dieterlen MT, Garbade J, Klein S, Dhein S, Mohr FW, Bittner HB. Identification of Non-HLA Antibodies in Ventricular Assist Device Recipients. J Heart Lung Transplant 2012;31:S46.

3. Dragun D, Muller DN, Brasen JH, Fritsche L, Nieminen-Kehla M, Dechend R, Kintscher U, Rudolph B, Hoebeke J, Eckert D, Mazak I, Plehm R, Schonemann C, Unger T, Budde K, Neumayer HH, Luft FC, Wallukat G. Angiotensin II type 1 receptor activating antibodies in renal-allograft rejection. N Engl J Med 2005;352:558-69.

4. Reinsmoen NL, Lai CH, Mirocha J, Cao K, Ong GD, Naim M, Wang Q, Riega S, Rafiei M, Patel J, Kobashigawa J. Increased Negative Impact of Anti-Angiotensin Type 1 Receptor Antibodies Together with De Novo Donor HLA Specific Antibodies on Graft Outcome in Heart Transplant Recipients. J Heart Lung Transplant 2013;32:S113.

5. Hiemann NE, Meyer R, Wellnhofer E, Schoenemann C, Heidecke $\mathrm{H}_{\text {, }}$ Lachmann N, Hetzer R, Dragun D. Non-HLA antibodies targeting vascular receptors enhance alloimmune response and microvasculopathy after heart transplantation. Transplantation 2012;94:919-24.

6. Kirklin JK, Naftel DC, Pagani FD, Kormos RL, Stevenson LW, Blume ED, Miller MA, Baldwin JJ, Young JB. Sixth INTERMACS annual report: A 10,000-patient database. J Heart Lung Transplant 2014;33:555-64.

7. Drakos SG, Stringham JC, Long JW, Gilbert EM, Fuller TC, Campbell BK, Horne BD, Hagan ME, Nelson KE, Lindblom JM, Meldrum PA Carlson JF, Moore SA, Kfoury AG, Renlund DG. Prevalence and risks of allosensitization in HeartMate left ventricular assist device recipients: the impact of leukofiltered blood product transfusions. Thorac Cardiovasc Surg 2007;133:1612-19.

8. McKenna DH, Eastlund T, Segall M, Noreen HJ, Park SJ. HLA alloimmunization in patients requiring ventricular assist device support. J Heart Lung Transplant 2002;21:1218-24.

9. Massad MG, Cook DJ, Schmitt SK, Smedira NG, McCarthy JF, Vargo RL, McCarthy PM. Factors influencing HLA sensitization in implantable LVAD recipients. Ann Thorac Surg 1997;64:1120-25.

10. Barten MJ, Dragun D, Dieterlen MT, Salisch Sv, Garbade J, Klein S,
Dhein S, Mohr FW, Bittner HB. Non-HLA Antibody Screening after Heart Transplantation Identifies High Risk for Cardiac Allograft Vasculopathy. J Heart Lung Transplant 2012;31:S107.

11. Dragun D. The role of angiotensin II type 1 receptor -activating antibodies in renal allograft vascular rejection. Pediatr Nephrol 2007;22:911.

12. Dechend R, Homuth V, Wallukat G, Kreuzer J, Park JK, Theuer J, Juepner A, Gulba DC, Mackman N, Haller H, Luft FC. AT(1) receptor agonistic antibdies from preeclamptic patients cause vascular cells to express tissue factor. Circulation 2000;101:2382-7.

13. Xia Y, Zhou CC, Ramin SM, Kellems RE. Angiotensin receptors, autoimmunity, and preeclampsia. J Immunol 2007;179:3391-5.

14. Yang S, Zhong Q, Qiu Y, Chen X, Chen F, Mustafa K, Ding D, Zhou Y, Lin J, Yan S, Deng Y, Wang M, Zhou Y, Liao Y, Zhou Z. Angiotensin II receptor type 1 autoantibodies promote endothelial microparticles formation through activating p38 MAPK pathway. J Hypertens 2014;32:762-70.

15. Zhang SL, Du YH, Wang J, Yang LH, Yang XL, Zheng RH, Wang YWK, Zhang MS, Liu HR. Endothelial dysfunction induced by antibodies against angiotensin AT1 receptor in immunized rats. Acta Pharmacol Sin 2010;31:1381-8.

16. Du Q, Wu J, Wang H, Wang X, Xu L, Zhang Z, Liu J, Zhang J, Chen J, Hakonarson $\mathrm{H}, \mathrm{Hu}$ A, Zhang L. Perindopril treatment promote left ventricle remodeling in patients with heart failure screened positive for autoantibodies against angiotensin II type 1 receptor. BMC Cardiovasc Disord 2013;13:94.

17. Dragun D, Philippe A, Catar R. Role of non-HLA antibodies in organ transplantation. Curr Opin Organ Transplant 2012;17:440-5.

18. Win TS, Pettigrew GJ. Humoral autoimmunity and transplant vasculopathy: when allo is not enaugh. Transplantation 2010;90:113.

19. Mogi M, Iwai M, Horiuchi M. New insighs into the regulation of angiotensin receptors. Curr Opin Nephrol Hypertens 2009;18:138-43.

20. van Geel PP, Pinto YM, Voors AA, Buikema H, Oosterga M, Crijns HJ, van Gilst WH. Angiotensin II type 1 receptor A1166C gene polymorhism is associated with an incresed response to angiotensin II in human arteries. Hypertension 2000;35:717-21.

21. Wu J, You J, Wang S, Zhang L, Gong H, Zou Y. Insights into the activation and inhibition of Angiotensin II Type 1 Receptor in the mechanically loaded heart. Circ J 2014;78:1283-9.

22. Storch U, Mederos Y, Schnitzler M, Gudermann T. G protein-mediated stretch reception. American Journal of Physiology. Am J Physiol Heart Circ Physiol 2012;302:H1241-9.

23. Dragun D, Hegner B. Non-HLA antibodies post-transplantation: clinical relevance and treatment in solid organ transplantation. Contrib Nephrol 2009;162:129.

24. Kaminska D, Tyran B, Mazanowska O, Rabczynski J, Szyber P, Patrzalek $D$, Chudoba P, Polak W, Klinger M. Cytokine gene expression in kidney allograft biopsies after donor brain death and ischemia-reperfusion injury using in situ reverse-transcription polymerase chain reaction analysis. Transplantation 2007;84:1118. 